\title{
Tension-induced vesicle fusion: pathways and pore dynamics
}

\author{
Lianghui Gao, ${ }^{* a b}$ Reinhard Lipowsky ${ }^{b}$ and Julian Shillcock $\dagger^{b}$ \\ Received 25th January 2008, Accepted 10th April 2008 \\ First published as an Advance Article on the web 24th April 2008 \\ DOI: 10.1039/b801407h
}

The dynamics of tension-induced fusion of two vesicles is studied using dissipative particle dynamics (DPD) simulations. The vesicle membranes use an improved DPD parameter set that results in their sustaining only a $10-30 \%$ relative area stretch before rupturing on the microsecond timescale of the simulations. Two distinct fusion pathways are observed depending on the initial vesicle tensions. In pathway I, at low membrane tension, a flattened adhesion zone is formed between the vesicles, and one vesicle subsequently ruptures in this contact zone to form a hemifusion state. This state is unstable and eventually opens a pore to complete the fusion process. In pathway II, at higher tension, a stalk is formed during the fusion process that is then transformed by transmembrane pore formation into a fusion pore. Whereas the latter pathway II resembles stalk pathways as observed in other simulation studies, fusion pathway I, which does not involve any stalk formation, has not been described previously to the best of our knowledge. A statistical analysis of the various processes shows that fusion is the dominant pathway for releasing the tension of the vesicles. The functional dependence of the observed fusion time on membrane tension implies that the fusion process is completed by overcoming two energy barriers with scales of $13 k_{\mathrm{B}} T$ and $11 k_{\mathrm{B}} T$. The fusion pore radius as a function of time has also been extracted from the simulations, and provides a quantitative measure of the fusion dynamics which are in agreement with recent experiments.

\section{Introduction}

Membrane fusion is essential to the life of eukaryotic cells. Cells communicate with the world around them through vesicle-based transport. Such transport vesicles bud off from one membrane and fuse with another. Exocytosis, protein trafficking, fertilization, and viral infection are all based on this fundamental process. ${ }^{1}$ In biological systems, the process of membrane fusion is accomplished with the aid of proteins: ${ }^{2}$ first, targeting proteins bring the fusing membranes into close contact. Then a fusion pore forms. The fusion pore may be composed only of fusion proteins, or a combination of proteins and lipids, or only lipids. The presumably simplest way to induce fusion is via membrane tension arising from the osmotic conditions as has been studied experimentally, e.g., in ref. 3,4. In addition, membrane fusion can be induced by the adsorption of cations ${ }^{5}$ and by polymers such as $\mathrm{PEG}^{6}$ which may induce gradients in membrane tension. ${ }^{7}$ In this paper, we focus on the mechanism and dynamics of tensioninduced membrane fusion.

Limited by the length and time resolution, the formation of the fusion pore cannot yet be visualized experimentally. In general, the formation of the fusion pore must be preceded by local contacts between the two membranes which may lead to pointlike defects, so-called stalks, in which the proximal monolayers of the two bilayers are connected whereas the two distal monolayers

\footnotetext{
aDepartment of Chemistry, Beijing Normal University, Beijing 100875 , China.E-mail:lhgao@bnu.edu.cn

${ }^{b}$ Max Planck Institute of Colloids and Interfaces, Potsdam 14424, Germany.E-mail: lipowsky@mpikg.mpg.de

$\dagger$ Present address: MEMPHYS, University of Southern Denmark, Campusvej 55, DK-5230 Odense M, Denmark.
}

are still separated. ${ }^{8,9}$ Several variants of such stalks have been described and their energies calculated on the basis of continuum elasticity theory. ${ }^{10-13}$ In one proposed pathway, ${ }^{10}$ the stalk expands radially to form a hemifused state in which the distal monolayers touch but the aqueous contents are still separated. This hemifusion state eventually ruptures to complete the fusion process. In another pathway, ${ }^{11}$ rather than expanding radially, the appearance of the stalk is followed quickly by the formation of a transmembrane pore crossing both the contacting bilayers. Such a fusion pore opening is referred as direct fusion.

In general, continuum elastic models for membrane fusion are based on simplifying assumptions about certain intermediate membrane states such as stalks. In contrast, computer simulations can be used to obtain unbiased information on the detailed fusion pathways. But all-atom MD simulations are limited to short times and small system sizes by their complex force fields and small integration step size. ${ }^{14}$ Thus coarse grained (CG) methods have been developed to investigate the dynamics of membrane fusion on more realistic length and time scales. Noguchi and Takasu ${ }^{15,16}$ employed solvent-free Brownian dynamics simulations and found that besides the direct fusion pathway, which is assumed by the continuum stalk model, ${ }^{11}$ an alternative pathway exists in which an elliptic stalk appears and contact between the ends of the banana-shaped stalk leads to a pore opening. Such a stalk mechanism was also observed by Stevens et al. ${ }^{17}$ in coarse grained MD simulations. Monte-Carlo simulations, ${ }^{18}$ self-consistent field theory, ${ }^{19,20}$ and CG MD simulations ${ }^{21}$ also predict another possible fusion pathway: in the vicinity of a stalk, a transient (or leaky) hole forms. Holes in each bilayer preferentially nucleate next to the same stalk. The stalk propagates along the edges of the aligned holes to complete the fusion process. Such an intermediate may be identical to that 
described in Ref. 15-17, but the latter works did not postulate that fusion is leaky.

Recently dissipative particle dynamics (DPD) simulations have been used to study membrane fusion events ${ }^{22,23}$ as the method can reach longer length and time scales than MD simulations for a given computational cost. The main advantage of using DPD instead of coarse-grained MD is its speed and computational cheapness. Although coarse-grained MD provides a more accurate chemical representation of the component molecules, we believe that such detail is not essential for understanding generic aspects of collective phenomena such as tension-driven membrane fusion. As has just been mentioned, several distinct types of simulation model predict similar mechanisms for tension-driven fusion. What these models have in common is that they capture the essential physical features of the constituent molecules (their amphiphilic nature), place these molecules in a well-defined state (vesicles or planar bilayers under tension), and follow their rearrangement under the action of forces that have been calibrated separately to ensure the membranes reproduce the gross behaviour of the experimental systems. The latter point usually means that the area per molecule and elastic moduli of the membranes have been adjusted to the appropriate experimental values. For DPD model membranes, the phase diagrams of lipid bilayers have been extensively explored by Smit et al., ${ }^{24}$ and recent reviews have compared membrane properties obtained from various types of coarse-grained simulation techniques, finding good agreement between them. ${ }^{25,26} \mathrm{~A}$ global morphology diagram of tensioninduced fusion of a vesicle and planar membrane patch has been constructed. ${ }^{22}$ It was found that the initial tension can be released by several alternative pathways: hemifusion, fusion and membrane rupture. Fusion events only occupy a small fraction of all the trials. In that work, the existence of pronounced hemifusion events showed that tension-induced membrane fusion in the simulations is not reliable. This effect is related to the fact that the simulated bilayers could resist up to $60 \%$ relative area stretch before rupture, whereas real lipid bilayers can only be stretched $5 \%$ before they burst.

In our recent work, ${ }^{27}$ we have systematically modified the DPD force parameters to simulate the self-assembly of a planar lipid bilayer in water. In our new model, an unporated fluid bilayer can resist $30 \%$ stretch before rupturing, and a porated bilayer reseals under $10 \%$ stretch, which brings the tense bilayer's dynamic properties closer to those of typical experimental lipid membranes, and very similar to those of polymersomes. ${ }^{28}$ In this article, we report the results of tension-driven fusion of vesicles modeled with our new DPD parameterization. ${ }^{27}$ For initial tensions sufficiently small that spontaneous membrane rupture requires a longer time than formation of a fusion pore, fusion is the most likely pathway for the vesicles to release their tension. Two kinds of fusion pathways are observed. Pathway $\mathrm{I}$ is observed at both high and low tension: the process begins by adhesion along a flattened contact zone. When the tension is large enough, one of the vesicles ruptures in the contact zone and a hemifused diaphragm forms. After some time, a pore appears that eventually opens to complete the fusion process. To the best of our knowledge, this fusion pathway I has not been described previously. Another fusion mechanism, pathway II, is observed at relatively high tension, and is consistent with the direct pathway given by the continuum model: ${ }^{11}$ a stalk forms at the center of two contacting vesicles, then a transmembrane pore nucleates and opens to complete fusion.

Fusion statistics collected under various initial membrane tensions show that fusion can always occur if the vesicles are subject to a relative area stretch between $5 \%$ and $25 \%$. The fusion time scale is defined as the time interval from kissing contact to the starting point of pore opening, and it decreases as the initial tension increases. It is found to vary from $2 \mu$ s to $100 \mathrm{~ns}$. We also study the radius of the fusion pore quantitatively, and find that the deviation of this radius from its asymptotic value for large times decays exponentially with time. The average speed of the expanding pore rim is of the order of $5 \mathrm{~cm} \mathrm{~s}^{-1}$, which is in excellent agreement with experiments. ${ }^{29}$

\section{Simulation method}

In coarse grained DPD simulations, ${ }^{30-34}$ pure water is modeled by soft beads with mass $m_{0}$ and diameter $r_{0}$. Each bead has a volume roughly equivalent to three water molecules. In our work, a lipid molecule is represented by three linearly-connected hydrophilic head beads with two hydrophobic tails attached to an adjacent pair. Each tail consists of four hydrocarbon beads. The interactions of the soft DPD beads are governed by short ranged repulsive forces with a prescribed combination of friction and random forces. Adjacent beads in a molecule are held together by a harmonic spring potential, and a bond-angle dependent potential is used to provide bending stiffness between triples of three consecutively bonded beads if desired. We note here that all the forces in the DPD simulations conserve momentum locally (including the thermostat forces), and this leads directly to the establishment of hydrodynamic modes in the fluids. Hydrodynamic forces may influence the fusion process as the viscosity of the aqueous medium acts as a frictional brake on the expansion of the fusion pore, affecting its time evolution. This viscosity is also present in $\mathrm{MD}$ simulations but requires much longer simulations to be established.

Our simulations are performed in the NVT ensemble. The derivation of an optimized DPD parameter set for lipid molecules in water, and the properties of the resulting lipid bilayer, are discussed in detail in our recent work. ${ }^{27}$ The same model membrane is used here, and its geometric and elastic properties (area per molecule, bilayer thickness and area expansion modulus and volume compressibility) are in agreement with experimental values for typical uncharged lipids such as dimyristoylphosphatidylcholine (DMPC). As described in ref. 27 , the length $r_{0}$ represents $0.76 \mathrm{~nm}$, and each DPD time step corresponds to $0.04 \mathrm{~ns}$ for a typical lipid such as DMPC. These length and time scales are used to convert the dimensionless simulation length and time scales to physical units.

To reduce the stretchability of the bilayer in the DPD simulations, a parallel and independent optimization of the force parameters has been done by Grafmüller et al. ${ }^{35}$ In that work, the basic strategy is to lower the repulsion force between tail beads. By doing so, the hydrophobic tails occupy a smaller area than the head groups which results in the positive spontaneous curvature of the monolayer. Then the bilayer is more easily ruptured. We have an independent parameter set that has been optimized as in Ref. 27 and apply it to study the fusion of two vesicles rather than 
one vesicle and one planar membrane patch. ${ }^{22,35}$ For two vesicles as studied here, we can monitor and analyze the complete opening of the fusion pore up to its maximal size, which is determined by the area-to-volume ratio of the new, combined vesicle. For the fusion of a vesicle with a planar membrane segment as studied in Ref. 22 and 35, on the other hand, the late stages of the fusion pore opening will be affected by the periodic boundary conditions of the planar segment unless the linear size of this segment is sufficiently large compared to the vesicle diameter.

We study the fusion of two spherical vesicles which are identical in the sense that they have the same number of lipids and the same area. Initially, these two identical vesicles (with a diameter around $22 \mathrm{~nm}$ ), each containing 3040 amphiphiles, are placed in a simulation box of size $30 \mathrm{~nm} \times 30 \mathrm{~nm} \times 60 \mathrm{~nm}$ with a separation of about $2 \mathrm{~nm}$. The amphiphiles are distributed between the vesicles' inner and outer leaflets so that they have the same area per molecule, ensuring that the spontaneous curvature is close to zero. The remaining volume inside and outside the vesicles is filled with water. The total density $\rho$ of the beads is $3 / r_{0}^{3}$. The initial tension of the vesicles is determined by their area per lipid $A$ (or, equivalently, the radius of the vesicles). We showed in Ref. 27 that planar bilayers are almost tensionless for an area per lipid $A_{0}=1.12 r_{0}^{2} \approx 0.65 \mathrm{~nm}^{2}$. Here we assume that at this area the vesicles are also relaxed. With increasing $A$, the vesicles become tense. We use the relative area stretch (or strain) $\left(A-A_{0}\right) /$ $A_{0}$ as a control parameter rather than tension since the tension of a vesicle is not easy to calculate in the simulations. The vesicles typically rupture quickly for strains exceeding 30\%. Fusion events between vesicles subject to $7 \%, 12 \%, 16 \%, 20 \%$ and $25 \%$ strain are studied. For each fixed strain, 8 independent simulation runs are performed to give statistical averages.

\section{Results and discussions}

\subsection{Fusion pathways}

Initially, the two vesicles were placed close to each other with a separation of about $2 \mathrm{~nm}$. The fluctuation-induced repulsion between them can be overcome if their adhesion energy is sufficiently large, and they can form a flattened contact zone. The adhesion energy arises from the model lipids' head-head interactions, which are tuned to reproduce the experimental tendency for uncharged lipid bilayers to adhere when they are pushed together. This adhesion arises from van der Waals forces between the bilayers, and is enhanced for tense membranes by the hydrophobic attraction of the slightly-exposed lipid tails. Two initially relaxed vesicles do not fuse but only adhere during the whole DPD simulation time up to $4 \mu$ s. Even though the adhesion of the vesicles will typically increase the membrane tension, this tension is not sufficient to induce fusion of the vesicles: the relaxed vesicles maintain their integrity. Although we cannot exclude the possibility that vesicles with less than $5 \%$ area strain that appear to hemifuse will fuse at longer times than we are able to observe, a few runs of 8 microseconds (twice as long as any observed fusion event) did not reveal such a transition. We expect that as lipids flip-flop between the vesicles, and the disturbance to the bilayer structure relaxes over time, the tendency for the vesicles to fuse will decrease if they have not done so within a certain time.

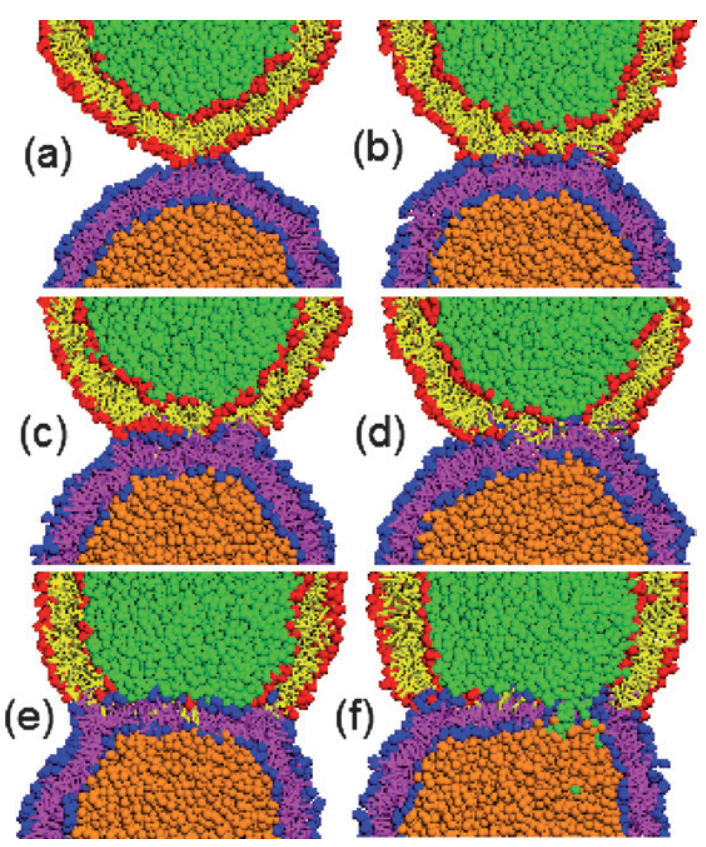

Fig. 1 Fusion pathway I for the low tension regime: time sequence of cross-sectional images for a typical fusion event of two vesicles under $16 \%$ relative area stretch. (a) Kissing contact at $t=700 \mathrm{~ns}$; (b) adhesion at $t=$ $960 \mathrm{~ns}$; (c) single-bilayer pore formation near the edge of the contact zone at $t=1040 \mathrm{~ns}$; (d) single-bilayer rupture at $t=1100 \mathrm{~ns}$; (e) hemifusion at $t=1360 \mathrm{~ns}$; (f) fusion pore opening at $t=1460 \mathrm{~ns}$. Coloring scheme: upper (lower) vesicle has red (blue) heads and yellow (purple) tails. Water inside the upper (lower) vesicle is green (gold). Water outside the vesicles is invisible for clarity.

Fusion occurs when the initial relative area stretch of the two vesicles exceeds 5 percent. A typical fusion process observed at $16 \%$ relative area stretch is shown in Fig. 1. After the vesicles make kissing contact at $700 \mathrm{~ns}$, they adhere over a large flat area. Such an adhesion state can persist for $300 \mathrm{~ns}$. Near the edge of the contact zone, due to the high curvature and thus the large local strain, the hydrocarbon chains can more easily splay and diffuse. The mixing of the lipids is observed preferentially near the edge of the contact zone by visual inspection of the snapshots of the fusing vesicles. Splayed lipids with one tail in each of the apposed monolayers were also seen by Stevens et al. ${ }^{17}$ who used MD simulations, which shows that similar molecular conformations arise in both coarse-grained MD and DPD fusion pathways. The adhesion-induced tension together with the initial tension can perturb the vesicles and trigger pore formation in one or both of them. Such pores often nucleate near the contact zone's edge and only breach one vesicle (different from the transmembrane pore in stalk models). ${ }^{10-13}$ If the pore in one vesicle forms within the contact zone, that vesicle ruptures and a hemifusion intermediate forms. This hemifusion diaphragm is mainly composed of lipids from the unruptured vesicle. In some cases, single-bilayer pores form in both of the individual vesicles and open at different locations. Then the hemifused bilayer has domains composed of lipids from both vesicles at the very beginning. The domains merge quickly via lateral diffusion of the lipids. This hemifused single bilayer finally ruptures near the edge of the contact zone to complete the fusion process. Compared to 
the previously-observed fusion events of a vesicle and a planar membrane patch in DPD simulations ${ }^{22}$ for which no decay of the hemifused state was observed over the whole simulation time of about 1-2 microseconds, this state decays after about 1.5 microseconds for the DPD parameter set used here. This fusion pathway I as described here is different (i) from the pathways predicted by continuum theory and (ii) from the ones observed in other simulation studies. No intermediate states in the form of stalks have been observed in our simulations. The fusion statistics show that for a large range of membrane tensions, vesicles adopt such a mechanism to fuse.

For larger membrane tensions, a direct fusion pathway, similar to that assumed by stalk models, ${ }^{11}$ is observed. Fig. 2 gives the snapshots of such a fusion process of two vesicles which are initially under $25 \%$ strain. Because the high tension allows the lipids in opposing bilayers to splay more easily, just after the vesicles contact with a small area, a highly curved and dimpled stalk quickly forms. Then a transmembrane, hydrophilic fusion pore forms across both of the membranes, and opens in a short time to allow water exchange. This second pathway can be regarded as a special case of pathway I. As a consequence of the high membrane tension, and thus smaller contact area, the single-bilayer pores in the individual vesicles are confined to a smaller region and easily align to form a single transmembrane pore. In this pathway II, hemifusion is skipped. The stalk as shown in Fig. 2(b) is a highly curved structure, which is similar to the one proposed within the modified continuum theory in Ref. 13. Within the latter theory, the energy of formation of this structure is estimated to be of the order of $40 k_{\mathrm{B}} T$. In contrast, we find that the characteristic energy barriers for the fusion process are of the order of $10 k_{\mathrm{B}} T$, see further below.

For membrane tensions close to the membrane rupture tension, for example vesicles under $30 \%$ strain, we have only observed two fusion events in 8 simulation runs, with one of them being leaky. In this leaky fusion event, a transient pore forms in one vesicle near the stalk and leaks water to the reservoir. After tens of ns, the pore reseals as the fusion pore opens. Such a pathway is similar to previous Monte Carlo simulation

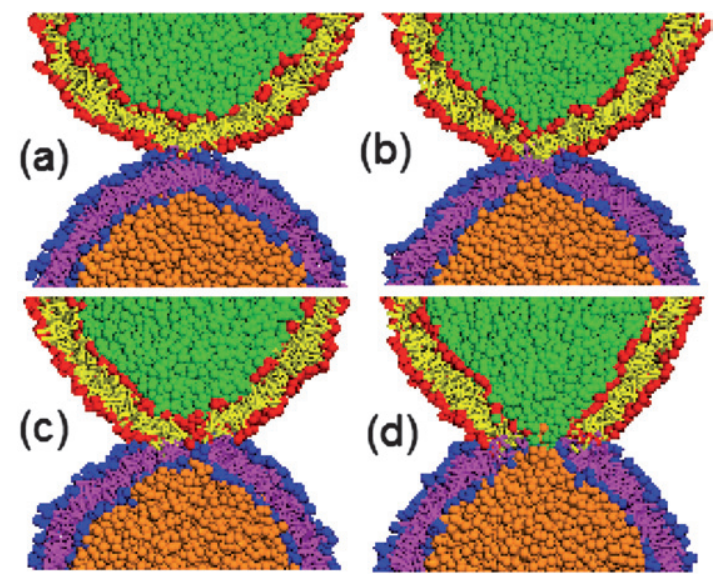

Fig. 2 Fusion pathway II for the high tension regime: time sequence of cross-sectional images for a direct fusion event of two vesicles under $25 \%$ relative area stretch. (a) Kissing contact at $t=260 \mathrm{~ns}$; (b) stalk formation at $t=320 \mathrm{~ns}$; (c) transmembrane fusion pore formation at $t=360 \mathrm{~ns}$; (d) fusion pore opening at $t=400 \mathrm{~ns}$. results. ${ }^{18}$ But under the same conditions, 6 other independent simulations show one or both of the vesicles rupturing before they get into contact. We conclude that such a leaky fusion process should be viewed as a special case of rupture, for which contact accidentally occurs earlier than rupture.

\subsection{Fusion statistics}

Fusion statistics are collected by running 8 independent simulations at a given strain (or, equivalently, tension). The results are listed in Table 1. When the initial strain of the fusing vesicles is larger than 10 percent, fusion is the final state of all the simulation runs except in several cases where the vesicles move apart without contact. In a previous study of membrane fusion between a vesicle and a planar membrane patch, ${ }^{22}$ in which the bilayers could resist $60 \%$ stretch, hemifusion was observed if the planar membrane was highly-stretched and the vesicle membrane more relaxed, whereas fusion (or membrane rupture) was observed if both membranes were initially tense. In our new model, as a consequence of the reduced membrane stretchability, hemifusion can only occur when the initial strains are around 5\%, and this end-state occupies only a small fraction of all the simulation trials. For initial strains between 5 and 10 percent, 3 hemifusion events and 4 fusion events are observed in 8 simulation runs with time up to $4 \mu$ s (in one trial the vesicles separated without contact). We propose that hemifusion is a metastable state rather than a stable state. As discussed in Ref. 27, the rupture tension is time dependent, and we expect that vesicles that hemifuse will eventually make a transition to complete fusion if sufficiently long simulations are run. When the strain is lower than $5 \%$, the two vesicles only adhere during the whole simulation time for all 8 runs.

\subsection{Fusion time scale}

We define the fusion time as the time interval between kissing contact of the two vesicles and the first appearance of the fusion pore. Because the vesicles fuse only under tension, it is highly unlikely that a pore reseals once it has formed in the adhered contact zone, so this definition is unambiguous; and because we exclude the pore expansion time from our definition, the fusion time only depends on local properties of the fusing membranes and not on the vesicle size. The pore expansion time by contrast depends strongly on the vesicle size, and is discussed in Section 3.4. The average fusion time $\left\langle t_{\mathrm{fu}}\right\rangle$ calculated from all the successful fusion events in Table 1 at each strain are plotted in Fig. 3. This time depends strongly on the initial strain (or tension). At $7 \%$ strain, it takes on average $2 \mu$ s for the vesicles to fuse, whereas the fusion time decreases to $200 \mathrm{~ns}$ for strains of

Table 1 Statistics of fusion events

\begin{tabular}{llllll}
\hline Strain & Adhesion & Hemifusion & Fusion & Rupture & Separation \\
\hline $0 \%$ & 8 & 0 & 0 & 0 & 0 \\
$7 \%$ & 0 & 3 & 4 & 0 & 1 \\
$12 \%$ & 0 & 0 & 6 & 0 & 2 \\
$16 \%$ & 0 & 0 & 7 & 0 & 1 \\
$20 \%$ & 0 & 0 & 7 & 0 & 1 \\
$25 \%$ & 0 & 0 & 7 & 0 & 1 \\
$30 \%$ & 0 & 0 & 2 & 6 & 0 \\
\hline
\end{tabular}




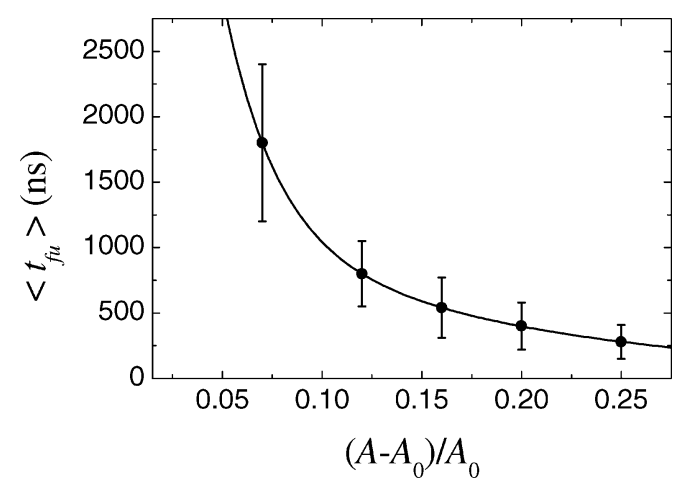

Fig. 3 Fusion time as a function of strain of the vesicles. The solid line represents the fit as provided by eqn (1).

$25 \%$. The average fusion time versus the strain can be well fitted by a double exponential function ${ }^{35}$

$$
\begin{gathered}
\left\langle t_{\mathrm{fu}}\right\rangle=t_{\mathrm{sc}}\left[\exp \left\{E_{1} / k_{\mathrm{B}} T-C_{1}\left(A-A_{0}\right) / A_{0}\right\}+\exp \left\{E_{2} / k_{\mathrm{B}} T-\right.\right. \\
\left.\left.C_{2}\left(A-A_{0}\right) / A_{0}\right\}\right]
\end{gathered}
$$

where $E_{1}$ and $E_{2}$ are the fusion energy barriers that two relaxed vesicles would need to overcome to fuse, $C_{1}$ and $C_{2}$ are fitting constants, and $t_{\mathrm{sc}}=0.04 \mathrm{~ns}$ is one DPD time step. The fit in Fig. 3 gives the energy barriers $E_{1} \simeq 12.65 k_{\mathrm{B}} T, E_{2} \simeq 10.54 k_{\mathrm{B}} T$, and $C_{1}$ $\simeq 38.22, C_{2} \simeq 6.75$. For essentially tensionless membranes with $A=A_{0}$, the double exponential fit to the fusion time data as given by eqn (1) depends on the two energies $E_{1}$ and $E_{2}$ which we tentatively interpret as two different energy barriers. In the low tension regime, we found the fusion pathway I as shown in Fig. 1. Inspection of this figure suggests that the two energy barriers correspond to (i) poration of the bilayer of one adhering vesicle which is completed in Fig. 1(d) and (ii) poration of the hemifused bilayer completed in Fig. 1(f). This conjecture could be checked by performing further simulations in which the bead-bead conservative interactions are modulated so that the membrane ruptures at different tensions, and measuring the relative changes in the two energy barriers. If the second barrier height correlates with the observed rupture tension, as measured from a plot of membrane surface tension versus area per molecule, then our identification of the second barrier with the membrane rupture process is likely correct.

We also studied the fusion events of two smaller vesicles (1520 lipids in each vesicle) and two bigger vesicles (6080 lipids in each vesicle, data not shown). At fixed membrane tension, the fusion times for the smaller and bigger vesicles are within the statistical errors of our standard vesicle (3040 lipids per vesicle). Thus, in the present study, the fusion times seem to be fairly independent of the vesicle size while a strong size dependence was found by Grafmüller $e t$ al. ${ }^{35}$ Since fusion pathway I as observed here is different from the one observed in ref. 35 , such a different size dependence is conceivable but remains to be further clarified. In contrast to the fusion times, our simulations do imply a strong size dependence of the time required for the opening of the fusion pore as we will now discuss.

\subsection{Dynamics of fusion pore expansion}

Another quantity characterizing the fusion process is the time dependence of the pore radius. To measure the pore size, we
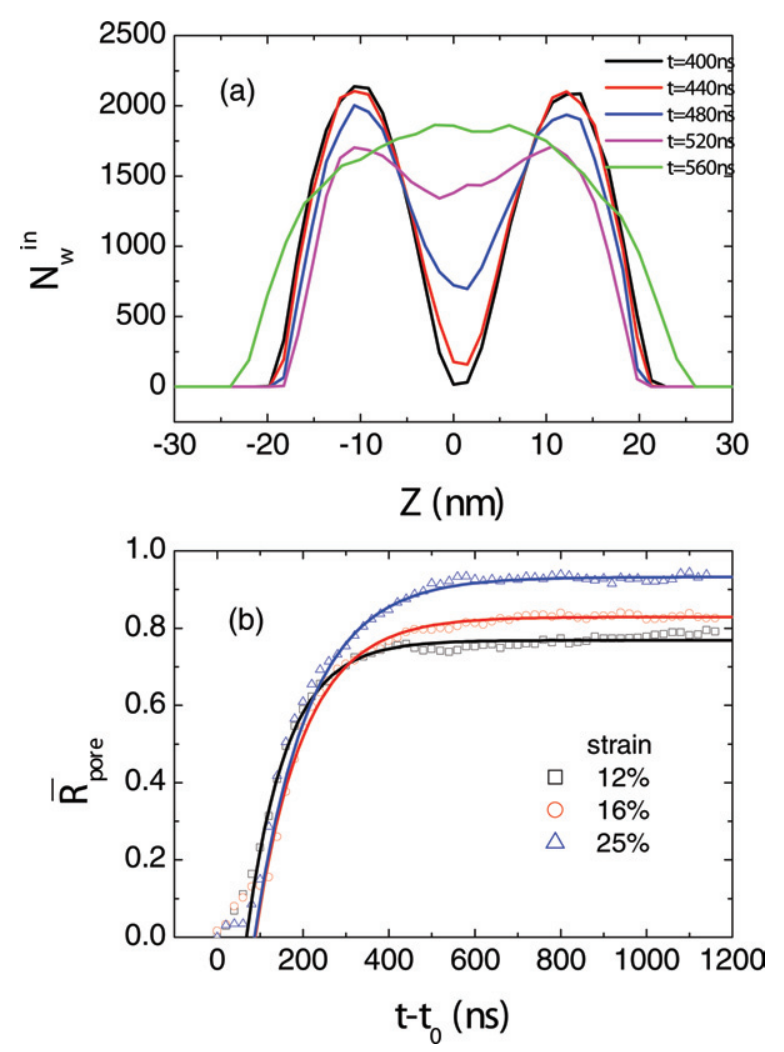

Fig. 4 (a) The distribution of water beads inside the vesicles as a function of the coordinate $Z$ parallel to the separation of the vesicle centers for different times $t$ and 25 percent relative area stretch (= strain). The curves correspond to the points in the time interval between $0 \mathrm{~ns}$ and $160 \mathrm{~ns}$ in Fig. 4(b). (b) The rescaled radius of the fusion pore as a function of time $t-t_{0}$, with $t_{0} 1800 \mathrm{~ns}, 1460 \mathrm{~ns}$, and $400 \mathrm{~ns}$ for vesicles with strain $12 \%, 16 \%$, and $25 \%$, respectively. Solid lines are the results fitted by eqn (2).

divide the simulation box into slices with thickness $\Delta L=2 r_{0} \approx$ $1.5 \mathrm{~nm}$ along the long axial direction $Z$ of the box. Then we count the number of water particles $N_{\mathrm{W}}^{\text {in }}(Z)$ that are initially inside the vesicles in each slice. Fig. 4(a) gives a sequence of curves showing the water distribution before and after the pore opening. The minimum value of each curve, which grows to a maximum value as the pores approach their equilibrium size, is designated as $N_{\mathrm{W}}^{\mathrm{pore}}$. This quantity gives a measure of the number of water particles in the fusion pore. Assuming that the pore has the shape of cylindrical disk, the radius of the fusion pore is given by $R_{\text {pore }}=\sqrt{N_{\mathrm{W}}^{\text {pore }} /(\pi \Delta L \rho)}$, with $\rho=3 / r_{0}^{3}$ being the bulk density of water particles in the simulation box. We define the rescaled radii of the pores, $\bar{R}_{\text {pore }}(t)=R_{\text {pore }}(t) / R_{\mathrm{V}}^{\text {in }}$, as a function of time $t-t_{0}$, and show its behavior for various initial vesicle tensions in Fig. 4(b). Here $R_{\mathrm{V}}^{\text {in }}$ is the inner radius of the vesicles before fusing, and $t_{0}$ is the time when the fusion pore starts opening. We find that the curves can be well fitted by an exponential function

$$
\bar{R}_{\text {pore }}(t)=\bar{R}_{\text {pore }}(\infty)\left[1-\exp \left(-\frac{t-t_{0}-t^{\prime}}{\tau}\right)\right]
$$

with the following three fitting parameters: the rescaled equilibrium pore size $\bar{R}_{\text {pore }}(\infty)$, pore opening time $t^{\prime}$, and pore relaxation time $\tau$. If the time $t_{0}$ obtained from the simulations is the 
actual time at which the pore opens, the parameter $t^{\prime}$ is zero. It thus quantifies the difference between the actual pore opening time obtained from fitting the pore size data, and the estimate obtained from inspecting images of the simulations. Here $t^{\prime}$ is $68 \mathrm{~ns}, 88 \mathrm{~ns}$, and $87 \mathrm{~ns}$ for vesicles with initial strain 12\%, 16\% and $25 \%$ respectively. Since at the very beginning of the pore opening, the assumption of a cylindrical pore shape fails, we did not include several data points close to $t_{0}$ when we fit the curves in Fig. 4(b).

The relaxation time $\tau$ is found to increase slowly as the initial vesicle tension increases. For example, $\tau$ is $93 \mathrm{~ns}, 100 \mathrm{~ns}$, and $125 \mathrm{~ns}$ for vesicles with initial strain $12 \%, 16 \%$ and $25 \%$ respectively. The increased relaxation time suggests that the vesicles adopt different fusion mechanisms for small and large tensions. For relatively low tensions, the fusion pore opening inside the hemifused bilayer is similar to the tension-induced rupture of a single bilayer. For relatively high tension, the fusion pore starts opening before a single-bilayer hemifusion state forms. The pore expands by pushing the whole fusion neck radically outwards, which is more difficult, and therefore slower than the rupture of a single bilayer. When the number of lipids in each vesicle is doubled (or the radius is increased by a factor of $\sqrt{ } 2$ ), the relaxation time $\tau$ goes up to $200 \mathrm{~ns}$ and $300 \mathrm{~ns}$ for vesicles with $16 \%$ and $25 \%$ strain respectively. Because of the limited ranges of the simulation box size and time that we can probe with current processor speeds, we are unable to measure quantitative finite size effects on $\tau$. But the dependence of the relaxation time for pore expansion on vesicle size may be qualitatively explained as follows. First, we note that the vesicles are impermeable to water on the time-scale of the fusion pore appearance and growth. Hence, they are under the constraint of constant volume. For the vesicles that fuse at $16 \%$ area strain, an increase in the vesicle radius of $\sqrt{ } 2$ leads to an increase in the vesicle area of a factor of two and so an increase in the adhered contact zone of a factor of two. As the pore expands by pushing aside the lipids in the contact zone, it takes longer for this process to occur in the larger vesicles. For vesicles that fuse at $25 \%$ area strain, the contact zone is initially smaller (see Fig. 2), and the pore expands by pushing apart the circular rim of fused vesicle membranes plus its surrounding ring of solvent. This takes proportionately longer than the lower-tension process of the pore expanding into the (constant area) contact zone.

The asymptotic pore radius ratio $\bar{R}_{\text {pore }}(\infty)$ extracted from Fig. 4(b) also increases with tension. Within the simulation time, at low tension, the final shape of the two fused vesicles is elliptic (or kinked as in Fig. 1(f) at even lower tension) with the long-time limit of the fusion pore radius being smaller than the initial vesicle radius. At high tension, the final state is more spherical with a fusion pore radius comparable to that of the initially highly tensed vesicle. The pore sizes at long times are not what one expects in an experiment. The prolate shape in experiment should be a sphere with $\bar{R}_{\text {ex }}(\infty)=2^{1 / 3}$. But the region in which the pore radius increases at constant speed is consistent with experiments. The averaged pore opening speed $R_{\text {pore }}(t) / \tau$, with $t=\tau+$ $t_{0}+t^{\prime}$, extracted from our simulations is of the order of $5 \mathrm{~cm} \mathrm{~s}^{-1}$ for all the fusion events regardless of the vesicles' size and initial tension. This average speed quantitatively agrees with the very recent experimental results where the vesicles have diameter around $50 \mu \mathrm{m},{ }^{29}$ and therefore is a good quantity that can be used to compare with real systems. Note, however, that the fusion of large vesicles is unlikely to proceed via the formation of a large hemifused diaphragm as observed in the simulations. Indeed, the observations in ref. 29 indicate a large diaphragm consisting of two bilayers connected by a fusion neck in the middle. Then the opening of this fusion neck, rather than the opening of the fusion pore, was measured and discussed in Ref. 29.

\section{Summary}

In this letter, DPD simulations of lipid bilayers are applied to investigate the dynamics of tension-induced membrane fusion of two vesicles. A DPD force parameter set is used that results in the vesicle membranes rupturing rapidly for strains around $30 \%$, which is an improvement on previous simulations in which the membranes could sustain up to $60 \%$ strain before rupturing. This change in the elastic response of highly-stretched membranes reveals different fusion pathways for the vesicles at low and high initial tensions. In one pathway, at relatively low tension, the fusion process proceeds via a flattened adhesion zone, then one vesicle ruptures within this contact zone and the adhered membranes hemifuse, followed by pore formation and subsequent expansion of the fusion pore. In the second pathway, at higher tension, fusion occurs through a process of stalk formation and radial opening of a transmembrane pore. The observed mean fusion time, defined as the interval from first contact of the vesicles to the fusion pore opening, decreases monotonically as the initial tension of the vesicles increases, and from which we also extract the fusion-energy barriers. The fusion pore expands exponentially with a relaxation time that depends only weakly on the initial tension. The average speed of the pore expansion is in agreement with recent experiments on much larger vesicles.

One important factor that affects the process of fusion is temperature which may speed up the fusion process as it makes lipid flip-flop more likely. But such effects are nontrivial. The temperature may first affect the structure and phase behavior of the lipid. We intend to investigate the role of temperature and the influence of lipid mixtures and domains in future studies.

\section{Acknowledgements}

We thank Andrea Grafmüller for stimulating discussions. MEMPHYS is supported by the Danish National Research Council

\section{References}

1 B. Alberts, A. Johnson, J. Lewis, M. Raff, K. Roberts, and P. Walter, Molecular Biology of the Cell, Garland Science, New York, 4th edn, 2002.

2 R. Jahn and H. Grubmüller, Curr. Opin. Cell Biol., 2002, 14, 488.

3 A. Finkelstein, J. Zimmerberg and F. S. Cohen, Annu. Rev. Physiol,, 1986, 48, 163.

4 A. Chanturiya, L. V. Chernomordik and J. Zimmerberg, Proc. Natl. Acad. Sci. U. S. A., 1997, 94, 14423.

5 K. Arnold, Structure and Dynamics of Membranes, Elsevier, 1995, vol. $1 \mathrm{~B}$.

6 J. Lee and B. R. Lentz, Proc. Natl. Acad. Sci. U. S. A., 1998, 95, 9274.

7 S. A. Safran, T. L. Kuhl and J. N. Israelachvili, Biophys. J., 2001, 81, 659.

8 D. Gingell and I. Ginsberg, in Membrane Fusion, ed. G. Poste and G. L. Nicolson, Elsevier, New York, 1978, p. 791. 
9 S. E. Hui, T. P. Stewart, L. T. Boni and P. L. Yeagle, Science, 1981, 212, 921 .

10 L. V. Chernomordik, G. B. Melikyan and Y. A. Chizmadzhev, Biochim. Biophys. Acta, 1987, 906, 309.

11 D. P. Siegel, Biophys. J., 1993, 65, 2124.

12 P. I. Kuzmin, J. Zimmerberg, Y. A. Chizmadzhev and F. S. Cohen, Proc. Natl. Acad. Sci. U. S. A., 2001, 98, 7235.

13 Y. Kozlovsky and M. M. Kozlov, Biophys. J., 2002, 82, 882.

14 S. Ohta-Iino, M. Pasenkiewicz-Gierula, Y. Takaoka, H. Miyagawam, K. Kitamura and A. Kusumi, Biophys. J., 2001, 81, 217.

15 H. Noguchi and M. Takasu, J. Chem. Phys., 2001, 115, 9547.

16 H. Noguchi, J. Chem. Phys., 2002, 117, 8130.

17 M. J. Stevens, J. H. Hoh and T. B. Woolf, Phys. Rev. Lett., 2003, 91, 188102.

18 M. Müller, K. Katsov and M. Schick, Biophys. J., 2003, 85, 1611.

19 K. Katsov, M. Müller and M. Schick, Biophys. J., 2004, 87, 3277.

20 G. J. A. Sevink and A. V. Zvelindovsky, Macromolecules, 2005, 38, 7502.

21 S. J. Marrink and A. E. Mark, J. Am. Chem. Soc., 2003, 125, 11144.

22 J. C. Shillcock and R. Lipowsky, Nat. Mater., 2005, 4, 225.
23 D. Li and X. Liu, J. Chem. Phys., 2005, 122, 174909.

24 M. Kranenburg, J. Nicolas and B. Smit, Phys. Chem. Chem. Phys., 2004, 6, 4142.

25 M. Venturoli, M. M. Sperotto, M. Kranenburg and B. Smit, Phys. Rep., 2006, 437, 1.

26 M. Mueller, K. Katsov and M. Schick, Phys. Rep., 2006, 434, 113.

27 L. Gao, J. C. Shillcock and R. Lipowsky, J. Chem. Phys., 2007, 126, 015101.

28 B. M. Discher, Y.-Y. Won, D. S. Ege, J. C.-M. Lee, F. S. Bates, D. E. Discher and D. A. Hammer, Science, 1999, 284, 1143.

29 C. K. Haluska, K. A. Riske, V. Marchi-Artzner, M. j. Lehn, R. Lipowsky and R. Dimova, Proc. Natl. Acad. Sci. U. S. A., 2006, 103, 15841.

30 P. J. Hoogerbrugge and J. M. V. A. Koelman, Europhys. Lett., 1992, 19, 155.

31 P. Espanol and P. Warren, Europhys. Lett., 1995, 30, 191.

32 R. D. Groot and P. B. Warren, J. Chem. Phys., 1997, 107, 4423.

33 R. D. Groot and K. L. Rabone, Biophys. J., 2001, 81, 725.

34 J. C. Shillcock and R. Lipowsky, J. Chem. Phys., 2002, 117, 5048.

35 A. Grafmüller, J. Shillcock and R. Lipowsky, Phys. Rev. Lett., 2007, 98, 218101. 